\title{
Publisher's Note: $\Delta(1232)$ effects in density-dependent relativistic Hartree-Fock theory and neutron stars [Phys. Rev. C 94, 045803 (2016)]
}

\author{
Zhen-Yu Zhu, Ang Li ${ }^{\circledR}$, Jin-Niu Hu, and Hiroyuki Sagawa
}

Q (Received 16 July 2020; published 23 July 2020)

DOI: 10.1103/PhysRevC.102.019905

This paper was published online on 21 October 2016 with the omission of a footnote label for the second author. Ang Li's footnote should read as "Corresponding author: liang@xmu.edu.cn". The paper has been corrected as of 20 July 2020 . The paper is incorrect in the printed version of the journal. 